$\underline{\xi}=-1$

\title{
Spatial Enhancement of AWiFS along Wider Swath using NSCT
}

\author{
K. S. R. Radhika ${ }^{1}$ C. V. Rao ${ }^{2}$, V. Kamakshi Prasad ${ }^{3}$ \\ ${ }^{1}$ Associate Professor, DMS SVH College Engineering, Machilipatnam. \\ ${ }^{2}$ Group Head, National Remote Sensing Centre, Hyderabad, India \\ ${ }^{3}$ Director of Evaluation, JNTUH, Hyderabad, India \\ *Corresponding Author Email: kammilisrr@gmail.com
}

\begin{abstract}
Image acquisition in a wider swath, cannot assess the best spatial resolution (SR) and temporal resolution (TR) simultaneously, due to inherent limitations of space borne sensors. But any of the information extraction from remote sensed (RS) images demands the above characteristics. As this is not possible onboard, suitable ground processing techniques need to be evolved to realise the requirements through advanced image processing techniques. The proposed work deals with processing of two onboard sensor data viz., Resourcesat-1 (RS1): LISS-III, which has medium swath combined with AWiFS, which has wider swath data to provide high spatial and temporal resolution at the same instant. LISS-III at $23 \mathrm{~m}$ and 24 days, AWiFS at $56 \mathrm{~m}$ and 5 days spatial and temporal revisits acquire the data at different swaths. In the process of acquisition at the same time, the $140 \mathrm{~km}$ swath of LISS-III coincides at the exact centre line $740 \mathrm{~km}$ swath of AWiFS. If the non-overlapping area of AWiFS has same features of earth's surface as of LISS-III overlapping area, it then provides a way to increase the SR of AWiFS to SR of LISS-III in the same non-overlapping area. Using this knowledge, a novel processing technique Fast One Pair Learning and Prediction (FOPLP) is developed in which time is optimized against the existing methods. FOPLP improves the SR of LISS-III in non-overlapping area using technique Single Image Super Resolution (SISR) with Non Sub sampled Contourlet Transforms (NSCT) method and is applied on different sets of images. The proposed technique resulting into an image having TR of 5 days, $740 \mathrm{~km}$ swath at SR of $23 \mathrm{~m}$. Results have shown the strength of the proposed method in terms of computation time and prediction accuracy assessment.
\end{abstract}

Keywords: Non Sub sampled Contourlet Transform, NSCT training and learning, Spatial Resolution, Temporal Resolution, LISS-III-AWiFS pair (One Pair), Single Image Super Resolution.

\section{Introduction}

Satellite data from various sensors is used in many applications like agriculture, defence, irrigation, surveillance, medical, traffic control, oceanography, etc. Fusion of images from multiple sensors resulting into enhancement of some of the resolutions of multi-resolution satellite images. Images acquired from different sensors give different information with their own specific characteristics. But different spectral bands information can be used for different application e.g., near infrared images give more information regarding vegetation, visible bands may give objects outline. When different types of images are fused combined information from different images can be obtained. India is agriculture based country and crops need to be monitored quite frequently. So images with high resolutions are required for these applications at more temporal revisits in nature. But the current satellite technology suffers with certain limitations and the problem could be overcome to certain extent, by constellation of satellites. Associated problem: it is an expensive option presently. For accurate follow up of agriculture, the crop study has to be closely monitored considering the variability of crops in temporally and also spatially.
For agricultural application this is important for decision making systems [8]. During monsoon agricultural lands are very much affected by floods [7]. Prediction of floods in advance, monitoring of natural hazards need data from wider swath, say for example, high TR and high SR data from total watersheds [1]. Watershed is a basic ecosystem. An ecosystem function over larger areas (entire ecosystems) is an invaluable integrated measurement and its functionality against several such systems is not an efficient assessment of environment as well as land cover. Upcoming ecological and land-cover variations can be estimated by temporal studies, for example, to evaluate the outcome of anthropogenic land-use routines on protected species [18]. Identifying different types of vegetation and habitats derivation require high spatial data for classification of land cover [9]. Wide swath images with high spatial and temporal resolution are needed in several applications of remote sensing e.g., accurate agricultural monitoring, prediction of floods, precision natural hazard follow up, total ecosystems and land cover land use variations.

In remote sensed sensors satellite program is offering a natural resource monitoring facility through a series of Resource Sat satellites (RS -1, 2, 2A). One of the specific advantage of this series is having a 3-tier sensor system, with varied resolutions at spatial, temporal, radiometric and swaths.

These missions also offer simultaneously similar spectral band widths and time of acquisition. These essential conditions facilitate in triggering the development of advanced techniques to 
meet the requirements of various information extraction. Linear Imaging Self Scanning Sensor (LISS-III) and Advanced Wide Field Sensor (AWiFS) are the two onboard sensors of ISRO's ResourceSat-1 Indian satellite. For LISS-III (High Resolution$\mathrm{HR}$ ), SR is $23 \mathrm{~m}$ and for AWiFS (Low Resolution-LR) it is $56 \mathrm{~m}$. LISS-III has temporal resolution of 24 days AWiFS 5 days respectively. Characteristics of the both sensors are given in Table-1.

Table 1: Characteristics of the sensors AWiFS and LISS-III of Indian satellite ResourceSat-1

\begin{tabular}{|c|c|c|c|c|}
\hline & \multicolumn{2}{|r|}{ LISS-III } & \multicolumn{2}{|r|}{ AWiFS } \\
\hline \multirow{4}{*}{$\begin{array}{c}\text { Spectral } \\
\text { Bands }\end{array}$} & B2 & $0.52-0.59 \mu \mathrm{m}$ & B2 & $0.52-0.59 \mu \mathrm{m}$ \\
\hline & B3 & $0.62-0.68 \mu \mathrm{m}$ & B3 & $0.62-0.68 \mu \mathrm{m}$ \\
\hline & B4 & $0.77-0.86 \mu \mathrm{m}$ & B4 & $0.77-0.86 \mu \mathrm{m}$ \\
\hline & B5 & $1.55-1.70 \mu \mathrm{m}$ & B5 & $1.55-1.70 \mu \mathrm{m}$ \\
\hline $\begin{array}{c}\text { Spatial } \\
\text { Resolution }\end{array}$ & & $23 \mathrm{~m}$ & & $56 \mathrm{~m}$ \\
\hline $\begin{array}{c}\text { Temporal } \\
\text { Resolution }\end{array}$ & & 24 days & & 5 days \\
\hline $\begin{array}{c}\text { Width of } \\
\text { Swath }\end{array}$ & & $140 \mathrm{~km}$ & & $740 \mathrm{~km}$ \\
\hline
\end{tabular}

However imaging at longer swath having high SR and TR at the same instant has been an impossible task with satellite RS systems. While ground sampling distance and swath width are directly related, revisit time is inversely related to swath width [15]. One more alternative to generate images with large swath at high SR and high TR is by improving the coarser SR to fine SR of the image using ground processing techniques. This is possible by using SISR [12]. SISR [2-5], initially put forth by Baker et al. 2002 SISR tries to obtain priori relationship used for amongst LR and HR image blocks and then applies this relationship to forecast HR image data from LR image data. SISR is put forth initially as a kernel learning concept and predicts the HR information by SVR (Support Vector Regression) [24-28].

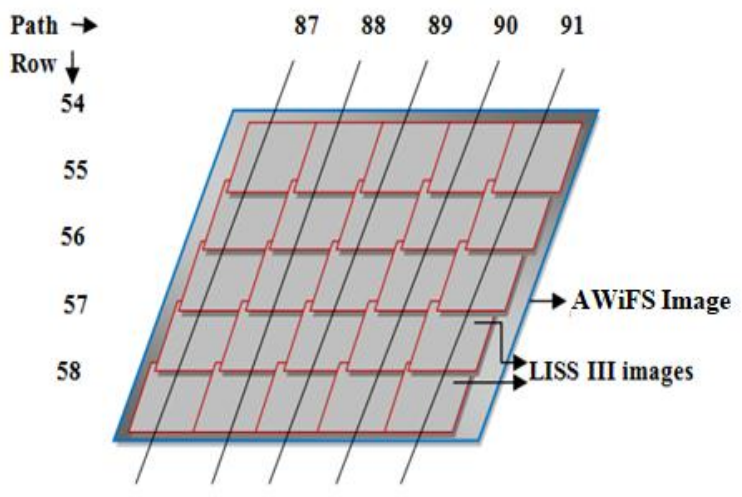

Fig. 1: AWiFS scene covering with images of LISS-III

In the Figure-1 the LR image is having a swath of $740 \mathrm{~km}$ with SR of $56 \mathrm{~m}$ is shown in bigger box outlined whereas the HR images have $23 \mathrm{~m} \mathrm{SR}$ and swath of $140 \mathrm{~km}$ are with smaller box outlines. Since the swath of LR image is $740 \mathrm{~km}$ we must mosaic $25 \mathrm{HR}$ images to cover the full scene of AWiFS. This is shown approximately in Figure-1. Suppose LR image is acquired at some date for example 30 Oct 09 at path 89 and row 56, LR image is also acquired at the same date and refered with same path and row. This scheme is designed to indicate the median times of respective sensor paths of LISS-III and AWiFS overlaps.

\section{Literature Study}

Baker and Kanade (2002) attempted to obtain matching of LR and HR image patches and later this result is used to acquire high resolution data using low resolution data, SISR serves as a kernel learning method and used to get HR information through SVR [24-28]. TR and SR of Landsat image are 16 days and $30 \mathrm{~m}$ where as for MODIS these are 1 day and $250 \mathrm{~m}-1000 \mathrm{~m}$. A pair of these two images is used to predict a fine image with a method called Location regularized sparse representation. Further fast iterative shrinkage thresholding algorithm was used for finding optimal solution [34].

On RS images SISR method [3-5] using SVR is applied to generate HR image [10]. In connection with pattern recognition concepts, Support Vector Machine (SVM) needed fewer number of samples for training [21-24]. Regression estimation used SVM and is referred as SVR [6]. SVR accepts an input of vector-valued of outputs a scalar valued. For every pixel, SVR model has to be trained with a group related HR pixel-LR patch pairs. HR pixel is obtained using the trained model with respective to the input LR patch. As its process of prediction is pixel-by-pixel, SVR fails to maintain the relationship with adjacent pixels though it has high prediction accuracy and requires least number of trained data samples. If prediction is done patch wise with overlapping of one or two pixels, relationship can be maintained with adjacent pixels. As a result, even there is a decrease in computational time [29].

Having a group of corresponding LR-HR patches in hand, HR image is generated from an LR image through SISR priori information is acquired from number of LR-HR pairs [11]. Input LR image and its respective HR image are not available in the above image pairs. Improvement of spatial resolution with swath expansion can be treated as SISR issue. This algorithm needs information regarding the required HR image priori information normalises the HR image which is obtained through prediction in SISR [20]. With regard to swath enhancement issue, before hand information is available only for the overlapped LISS-III image along with its respective AWiFS image. If same surface properties of earth are assumed between the non-overlapping portion of One Pair images overlapped area, in turn there is a way to improve the AWiFS SR with the LISS-III SR in the non-overlapped area. Bearing this in mind, priori information though limitedly in turn leads to a way to improve the SR of the non-overlapped area.

LR-HR local patches priori are possible from several number of LR-HR image pairs and provide training information to SISR technique. A suitable smoothing algorithm providing learning before improving the results is used by SISR technique [20]. In the proposed methodology, data for training related to LR-HR level patches are constrained to the regions of overlap between LISS-III image and its respective AWiFS image. Methodology presented here does not use any smoothness constraint as LR-HR local patches were obtained through contourlet domain. Contourlet transform has the strength to extract smoothness over the contours and simultaneously facilities learning the edge primitives and uses the training data related to LR-HR local patches above the contourlet domain [13].

Elaborate geometrical data about the image content is possible through multi resolution decomposition of an image in several directions [14]. Geometrical data provides greater degree of prediction accuracy during both training as well as prediction stages of local patches against multi resolution domain compared to spatial domain predicting patch wise information using overlap of one or more pixels (two) gives the way in rich neighbourhood relationship preserved with the adjoining pixels. Edges as well as adjoining pixels are significant to improve the SR of an image with SISR. Edges in multi resolution composition can be preserved by NSCT because of shift invariance property [19].

\section{Theoretical Basis}

\subsection{Non Subsample Contourlet Transforms}

Contourlet Transforms (CT) is suggested to describe 2D singularities related to image. Laplacian Pyramid (LP) and Directional Filter Bank (DFB) are contained in CT [17]. Though because of anisotropy, directionality CT describes curves scattered by, during decomposition and reconstructing an image with CT, frequency aliasing will be there. In view of decreasing frequency 
aliasing and improving directional selectivity along with shift invariance, NSCT which depends on Non Subsample Pyramid Decomposition and Non Sub sampled Filter Banks (NSFB) was proposed [19]. NSCT represents shift-invariant form of CT. Iterated non separable two channels NSFB is utilised by NSCT to get the shift invariance and nullify pseudo -Gibbs phenomenon round the singularities.

Along with multi resolution analysis, geometric and directional description is also given by NSCT [32]. NSCT overview is shown in Figure-2. 2D frequency plane is split to sub bands by filter banks contained in NSCT structure. Figure-2 shows frequency partitioning process. Due to reconstructing filter banks perfect reconstruction is possible for NSCT [16]. Compared to other methods of multi resolution analysis particularly with regard to image denoising and image enhancement, NSCT appears to be very efficient because of its multi scale, multi directionality, anisotropy and shift invariance properties [19].

Hence multi resolution decomposition is carried on RS images through NSCT to improve the SR using contourlet coefficient learning and prediction of HR image using this One Pair learning which is named as Fast One Pair Learning and Prediction (FOPLP).

\subsection{Fast One Pair Learning and Prediction}

This methodology uses Contourlet Coefficient Learning (CCL). Using property of shift invariance NSCT reduces an image to multiple direction, multiple level sub bands. Every sub band describes contourlet coefficients (CC).

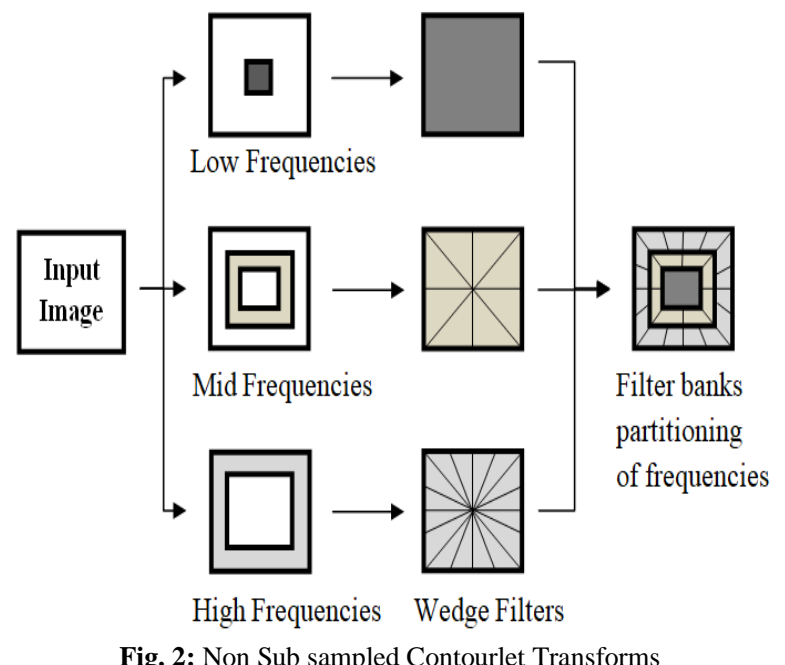

Training data information of the overlapped LISS-III image along with its respective AWiFS image are formed using CC. For each sub band, in the non-overlapping area, LISS-III pixels are predicted using AWiFS image using the training data information. CCL is the name to this process of learning coefficients with regard to CT domain.

Proposed methodology, FOPLP aims towards an ideal method to improve the SR using a SISR method against domain of NSCT training data are generated for every sub bands involving NSCT domain. CC of each patch is contained in the training data information and in turn correspondence exists between patches of One Pair. The results of the required HR patch for specific input LR patch obtained using suggested CCL for overlapping as well as non-overlapping areas of every sub band. Sub bands obtained through prediction in turn reconstructs the required image through inverse NSCT. The image resulted from reconstruction process will have HR image SR for overlapping as well as nonoverlapping areas of LR image.

\section{Methodology}

In Figure-1 it has been shown that the LISS-III and AWiFS images are acquired on the same date and same path and row. For the proposed work, FOPLP such images are considered to avoid atmospheric corrections, geometric errors, etc. Figure- 3 shows overlapping of $140 \mathrm{kms}$ swath corresponding to LISS-III at the center $740 \mathrm{~km}$ swath of AWiFS in acquisition process at the same instant [30]. Smaller swath (140km) of LISS-III can be extended to wider swath $(740 \mathrm{~km})$ provided same features of earth's surface are observed in non-overlapping area of AWiFS and LISS-III overlapping area. Overlapped LISS-III image patch and its respective AWiFS image patch in turn serve as prior knowledge to increase SR of non-overlapped area by FOPLP technique.

\subsection{Pre-processing of Image One Pair}

One Pair images must have identical geometry and of similar dimensions. To ensure this One Pair image data pixels should have one-to-one relation of correspondence between them. In geometric correction pixels of AWiFS image will be interpolated to the pixel size of LISS-III image. Hence one-to-one correspondence relationship is maintained to the pixels of both non-overlapping and overlapping areas in One Pair images. NSCT is applied to LISS-III overlapped portion with its respective to AWiFS image that is interpolated. Seven sub-bands are obtained for each image. These are the CC that is used in the training phase. Seven training databases are created for further prediction phase.

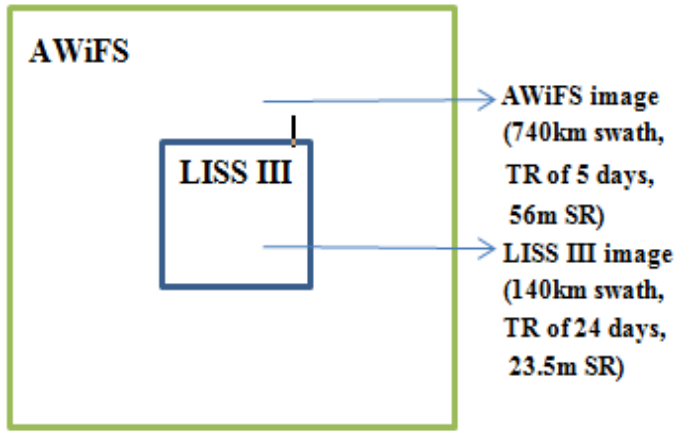

Fig. 3: Swath of One Pair images

\subsection{Creation of Training Databases from AWiFS and LISS-III Overlapped Area}

1. The entire scene of AWiFS is considered. The scene of LISS-III is overlapped at center of AWiFS image. One AWiFS image and one LISS-III image together is defined as One Pair. Consider the overlapped region of One Pair images.

2. NSCT is then applied to One Pair images separately. Applying NSCT uses decomposition in two levels. Decomposition in four directions is used for high level frequencies while decomposition is two directions is used for mid level frequencies. Low level contains the rest of the frequencies [19].

3. Each sub band is used to create the training database. Databases are created for individual sub band of both the LR and HR images.

4. Consider a $5 \times 5$ window in the overlapping region of One Pair images. Take one $5 \times 5$ patch from both the images of One Pair. These two patches are used for training.

5 . The window resets and then moves by one pixel to the right and so on. Once the window reaches column end, moves down to the next row. For the entire sub band this process is repeated. In every level, direction for each sub band and the data for training were thus generated.

6 . Obtained $5 \times 5$ patches are formed to $1 \times 25$ arrays in view of simplification of search and identifying process of patch of HR image with its respective patch of LR image.

ALGORITHM: Fast One Pair Learning and Prediction 
FAW $=$ Full AWiFS scene

$\mathrm{L} 1=$ Low pass sub band $\mathrm{CC}$ of AWiFS

M1, M2 = Middle pass sub band CC of AWiFS

$\mathrm{H} 1, \mathrm{H} 2, \mathrm{H} 3, \mathrm{H} 4=$ High pass sub band $\mathrm{CC}$ of AWiFS

$\mathrm{AP}=5 \times 5 \mathrm{AWiFS}$ patch

LP $=$ Best suited $5 \times 5$ LISS-III patch

$\mathrm{DB}=$ Database created in training for each CC

PL1 $=$ Predicted low pass sub band CC of AWiFS

PM1, PM2 = Predicted middle pass sub band CC of AWiFS

$\mathrm{PH} 1, \mathrm{PH} 2, \mathrm{PH} 3, \mathrm{PH} 4=$ Predicted high pass sub band $\mathrm{CC}$ of AWiFS

PFI $=$ Predicted image with SR as that of LISS-III and swath as that of AWiFS

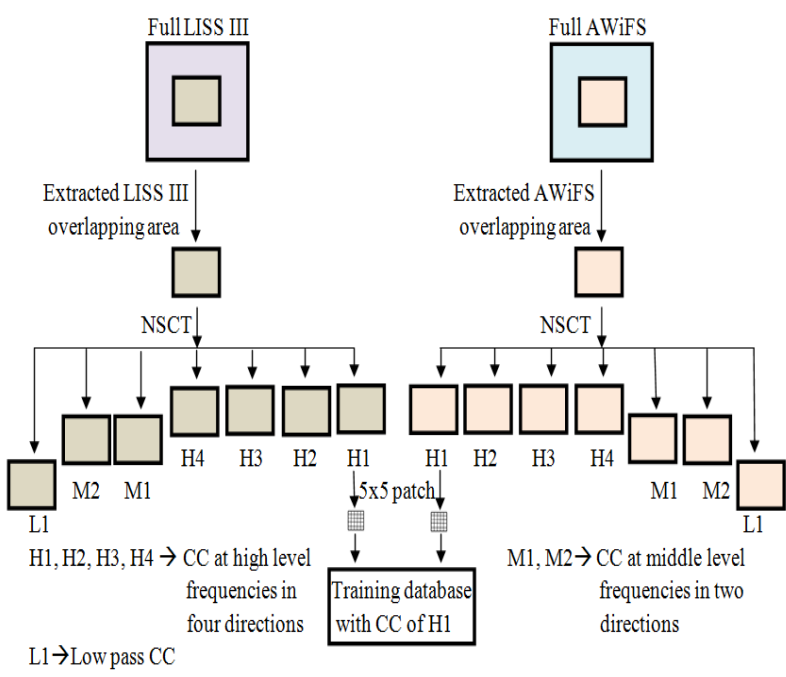

Fig. 4: In domain NSCT training of One Pair Images

The entire procedure explained through the above steps (1 to 6) is depicted in Figure-4. Creation of training database for H1 band is shown in the Figure. The entire procedure includes creation of training databases for all the remaining i.e. H2, H3, H4, M1, M2 and L1 (these are the CC's of the images).

\subsection{Prediction of HR Data for AWiFS Non-Overlapped Area}

1. Objective of the present study was to improve the SR of LR image in the non-overlapping area to the SR of HR image. Nonoverlapping as well as overlapping areas are present in the interpolated LR image. In the non-overlapping region to improve the SR the complete image serves as the input image.

2. Consider the full scene of the LR image for applying the NSCT. Here also the seven CC are obtained in different levels, directions. Again processing for prediction is done for each sub band separately.

3. In the training phase a $5 \times 5$ window is taken to consider the first LR patch. For this patch the best suited HR patch is found from the training database. Similarly window is moved to right and at the end moved to next row. The process is repeated with an overlap of one pixel and then averaged the one pixel overlap values. This process is repeated for all seven sub bands.

4. On the predicted sub bands the inverse NSCT is applied. Resulted predicted image is an HR image with resolution of LISSIII in the entire scene of AWiFS image.

5. Thus in non-overlapped region of AWIFS the SR is improved same as that of LISS-III SR. Thus synthetic image of AWiFS is generated with wider swath-740km, SR-23m and TR-5days.

The concept given through the steps (1 to 5) for obtaining swath expanded LISS-III image from the AWiFS image is shown in Figure-5. Steps for getting PL1 from L1 is only shown in the Figure and procedure includes similar implementation to M1, M2, $\mathrm{H} 1, \mathrm{H} 2, \mathrm{H} 3$ and H4 (CC's of image).

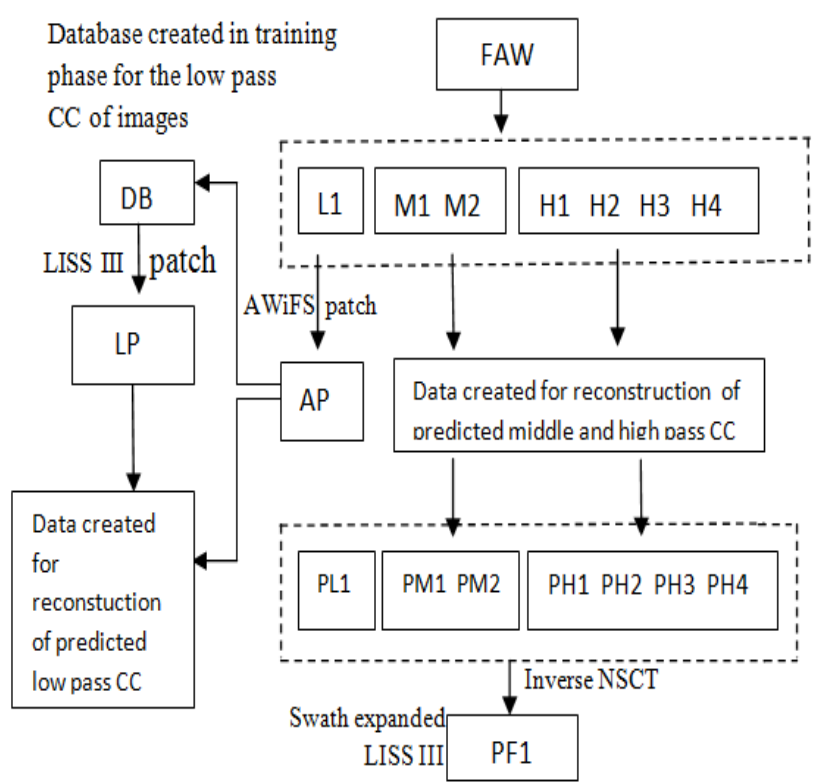

Fig. 5: High Resolution image construction using Fast One Pair Learning and Prediction algorithm

\section{Results}

The proposed method FOPLP is examined on different data sets taken at different areas. The experiments are conducted using the FOPLP method to improve the SR of AWiFS image to that of LISS-III image SR. To perform this input images are downloaded from BHUVAN website of National Remote Sensing Center [31]. While taking the One Pair images, it has been considered that both the images of One Pair are taken at same time and also at the same path and row. Size of 250 x 250 LISS-III image is considered and then it is degraded from which a simulated AWiFS image is created of $250 \times 250$ image. The overlapping region of HR and the LR simulated image have no differences spectrally, geometrically and radio metrically. Input images are considered in the southern parts of India. The dates of input images are considered in the crop season, so that it can be used for further studies. Our method FOPLP was compared with CCL method which is compared with SVR method. All the studies of both methods FOPLP and CCL are conducted on the same system.

In the One Pair images the overlapped area is taken with (x, y) coordinates as $(51,51)$ as the upper left corner and $(200,200)$ as the lower right corner. So the overlapped region is taken as $150 \mathrm{x}$ 150. In our method a neighbourhood patch of $5 \times 5$ is taken in the non-overlapping area starting at pixel location $(1,1)$. This patch is changed as $1 \times 25$ one dimensional array [p1, p2...p25]. The relevant LISS-III patch of this AWiFS patch is extracted from the training data base. This patch is used to reconstruct the specific pass bands. Then inverse NSCT is applied to all the sub bands to reconstruct the predicted image.

Quality assessment parameters are computed to analyse the quality of predicted image. Various performance measures have been proposed in the field of image fusion and also analysed the effects of fusion structures on the outcomes of fusion schemes. RMSE (Root Mean Squared Error), CC (Correlation Coefficient), $\mathrm{R}^{2}$ are some of the performance measures used to assess our predicted image quality with respect to the predicted one.

\subsection{Dataset I}

Test dataset is considered in the southern parts of India. It mainly covers many classes like water bodies, wetlands. Vegetation, barren soil, etc. 


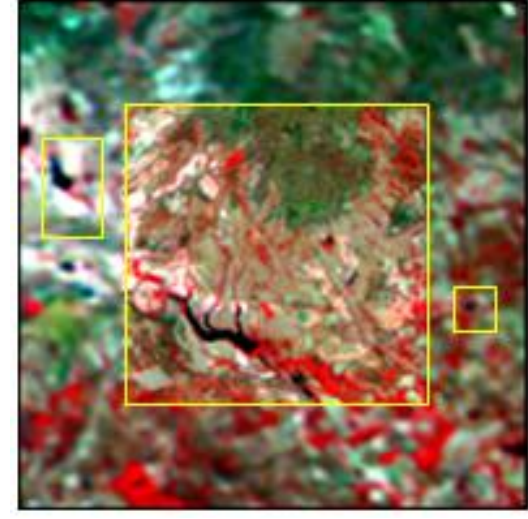

(a)

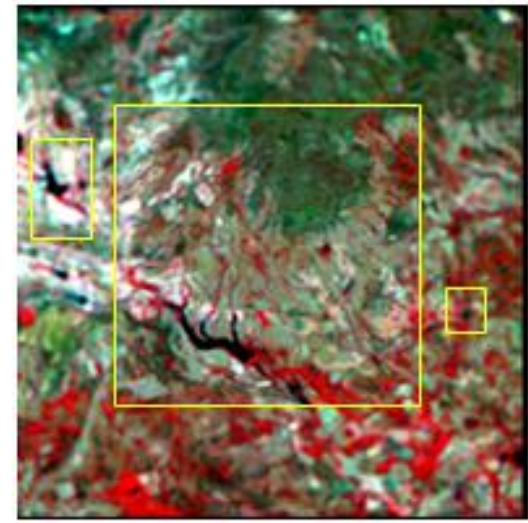

(b)

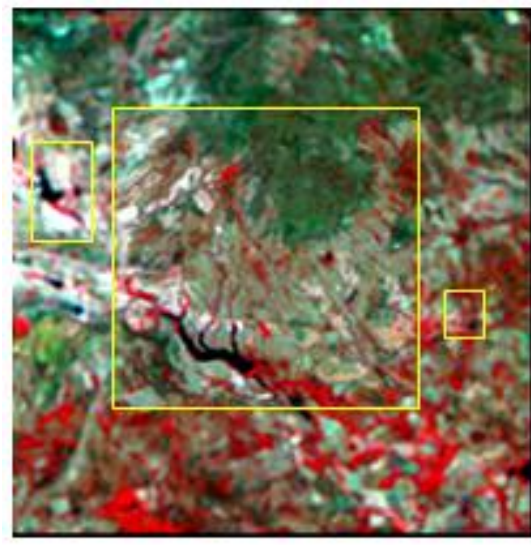

(c)

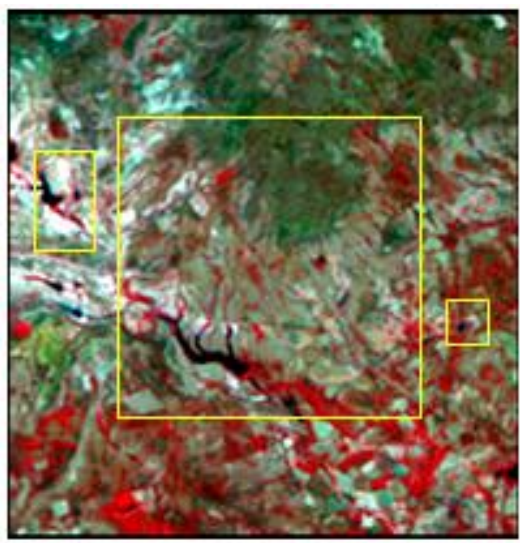

(d)

Fig. 6: HR image predicted with larger swath using CLL and FOPLP methods. [a]. Full scene of LR and HR at center. [b]. CCL predicted image. [c]. FOPLP predicted image. [d] HR image original
Figure 6a shows the input simulated LR image on which the HR image is overlapped at center of the LR image. Figure $6 b$ is the CCL methods output image and Figure $6 c$ is the predicted image from the FOPLP algorithm. Figure $6 \mathrm{~d}$ is the original HR image. All the images are shown in FCC (False Colour Composite) of B2, B3, B4 i.e. Green band, Red band, NIR band.

Table 2: Computational time in seconds for Dataset I

\begin{tabular}{|c|c|c|}
\hline Method & $\begin{array}{c}\text { FOPLP(Proposed } \\
\text { Method) }\end{array}$ & $\begin{array}{c}\text { CCL(Existing } \\
\text { Method) }\end{array}$ \\
\hline Band 2 & 5234 & 17224 \\
\hline Band 3 & 5314 & 17541 \\
\hline Band 4 & 5475 & 17254 \\
\hline Band 5 & 5313 & 17486 \\
\hline
\end{tabular}

Table 3: Quality assessment parameters for Dataset I

\begin{tabular}{|c|c|c|c|}
\hline $\begin{array}{c}\text { Quality } \\
\text { Assessment } \\
\text { Parameter }\end{array}$ & RMSE & $\mathbf{C C}$ & $\mathbf{R}^{\mathbf{2}}$ \\
\hline Band 2 & 0.0041 & 0.9679 & 0.9322 \\
\hline Band 3 & 0.0068 & 0.9678 & 0.9305 \\
\hline Band 4 & 0.0130 & 0.9402 & 0.8988 \\
\hline Band 5 & 0.0082 & 0.9776 & 0.9546 \\
\hline
\end{tabular}

Table-2 describes how strong our method is regarding the computational time for each band compared with that of CCL method. There is lot of difference in computational time between the CCL and FOPLP method. Table-3 shows the different quality assessment parameters between the predicted and the original images.

\subsection{More Datasets}

The proposed method FOPLP is checked on some more datasets proved that it has given better results mainly optimized in time.

Table 4: Computational time in seconds

\begin{tabular}{|c|c|c|c|c|c|c|}
\hline & \multicolumn{2}{|c|}{ Dataset II } & \multicolumn{2}{c|}{ Dataset III } & \multicolumn{2}{c|}{ Dataset IV } \\
\cline { 2 - 7 } & FOPLP & CCL & FOPLP & CCL & FOPLP & CCL \\
\hline B2 & 5141 & 17859 & 5140 & 17628 & 5119 & 17627 \\
\hline B3 & 5147 & 17888 & 5142 & 17366 & 5104 & 17620 \\
\hline B4 & 5144 & 17990 & 5272 & 17854 & 5115 & 17964 \\
\hline B4 & 5193 & 17486 & 5377 & 17472 & 5106 & 17720 \\
\hline
\end{tabular}

Table 5: Quality assessment parameters

\begin{tabular}{|c|c|c|c|c|}
\hline & & RMSE & CC & $\mathbf{R}^{\mathbf{2}}$ \\
\hline \multirow{4}{*}{ Dataset II } & B2 & 0.0064 & 0.9516 & 0.8835 \\
\cline { 2 - 5 } & B3 & 0.0100 & 0.9465 & 0.8753 \\
\cline { 2 - 5 } & B4 & 0.0257 & 0.8904 & 0.7821 \\
\cline { 2 - 5 } & B5 & 0.0340 & 0.9559 & 0.8900 \\
\hline \multirow{7}{*}{ Dataset III } & B2 & 0.0018 & 0.9898 & 0.9796 \\
\cline { 2 - 5 } & B3 & 0.0085 & 0.9705 & 0.9418 \\
\cline { 2 - 5 } & B4 & 0.0143 & 0.9105 & 0.8089 \\
\cline { 2 - 5 } & B5 & 0.0090 & 0.9732 & 0.9420 \\
\hline \multirow{7}{*}{ Dataset IV } & B2 & 0.0053 & 0.9638 & 0.9411 \\
\cline { 2 - 5 } & B3 & 0.0093 & 0.9637 & 0.9550 \\
\cline { 2 - 5 } & B4 & 0.0167 & 0.9593 & 0.9331 \\
\cline { 2 - 5 } & B5 & 0.0114 & 0.9788 & 0.9698 \\
\hline
\end{tabular}

Table-4 describes how strong is our method regarding the computational time that of CCL method. Table-5 shows the different quality assessment parameters of predicted image.

\section{Discussions}

The time of our proposed method is almost three times less than that of CCL method. If the image size is larger, the computation time changes in exponential order. In CCL method, computed the RMSE error with all patches that are there in the training database and sorted for further processing. In our work the best patch is found in an optimized method. If a relevant patch is found with an error of zero then no processing is done and this patch is considered as best patch. If non overlapping area has similar features as that of overlapping area then same patch is found in the 
training database. This patch is considered as the best patch for the testing patch. The AWiFS image swath is $740 \mathrm{~km}$ which is obtained by two sensors namely AWiFS - A and AWiFS - B. Both sensors acquire each $370 \mathrm{~km}$ swath. Since the One Pair images are having no differences spectrally and radio metrically so the LISS-III swath is expanded for $740 \mathrm{~km}$. Further the temporal resolution of LISS-III can also be acquired as that of AWiFS image. The proposed method has been evalued with simulated LR image and the HR original. The results have been validated and proved to be satisfactory.

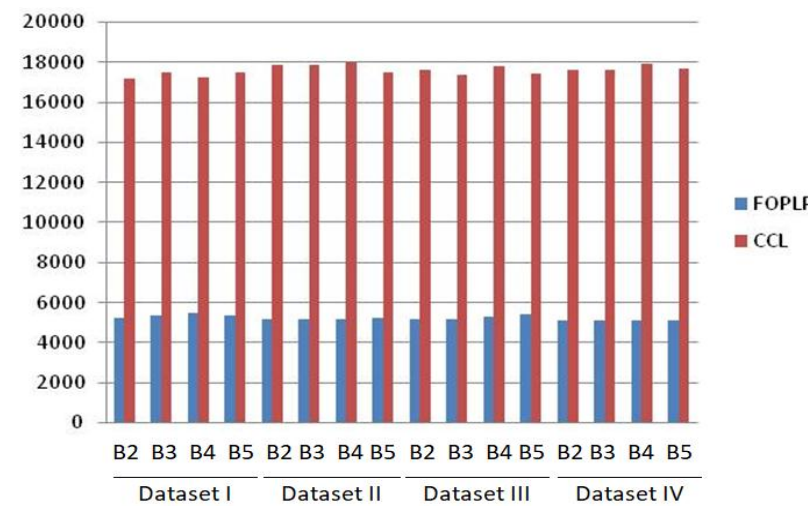

Fig. 7: Computation Time Comparison

As seen from the comparison plots, Figure-7, the computation time for the proposed method of FOPLP is relatively less than that of the existing CCL method. SVR and CCL method used the technique SISR for the spatial improvement of AWiFS image. SVR method predicted the HR image using pixel wise so relationship between the neighbouring pixels is not maintained [10]. CCL method predicted HR image patch wise with less time than the SVR method [29]. Our method FOPLP predicted the HR image using One Pair images which is proved to be satisfactory regarding the quality assessment parameters and also has taken less computational time than the above methods.

\section{Conclusion}

Enhancement of spatial resolution was examined on different datasets. Therefore AWiFS image is predicted with spatial resolution $-23.5 \mathrm{~m}$, swath $-740 \mathrm{~km}$ and 5 days revisit time. Improvement of spatial resolution of AWiFS image is investigated and the quality assessment parameters are proved to be satisfactory assuming that features on the surface of the Earth in the overlapping area of the One Pair images may also be contained in the AWiFS non overlapping region. Thus AWiFS spatial resolution is improved as that of LISS-III spatial resolution. Our method used SISR technique assuming that it has LISS-III image prior knowledge. The proposed method FOPLP is having detailed information only in the overlapped region of One Pair, but has been predicted the HR patch corresponding to the LR patch more accurately and faster than CCL and SVR methods. Thus a synthetic HR image is created with characteristics of 5 days TR, $23.5 \times 23.5 \mathrm{~m} \mathrm{SR}$ and swath of $740 \mathrm{~km}$. Since many satellites are having a limitation of acquiring high resolution in all respects like SR, TR and swath simultaneously. Images with high SR along wider swath can be obtained by our method. Even though our method outperforms the existing methods regarding the computational time still future work is required to improve the TR with more accuracy in prediction, reduce the time of computation.

\section{References}

[1] ASAR Wide Swath and Image Mode Data in Agricultural Areas." IEEE Transactions on Geosciences and Remote Sensing 44: 889899. doi:10.1109/TGRS.2005.863858.
[2] Baker, S., and T. Kanade. 2002. "Limits on Super-Resolution and How to Break Them." IEEE Transactions on Pattern Analysis and Machine Intelligence 24: 1167-1183. doi:10.1109/ TPAMI. 2002.1033210

[3] Freeman, W. T., T. R. Jones, and E. C. Pasztor. 2002. "ExampleBased Superresolution." IEEE Computer Graphics and Applications 22: 56-65. doi:10.1109/38.988747.

[4] Chang, H., D. Y. Yeung, and Y. Xiong. 2004. "Super-Resolution through Neighbor Embedding." Proceedings IEEE Conference CVPR 1: I-275-I-282.

[5] Elad, M., and D. Datsenko. 2009. "Example-Based Regularization Deployed to Super-Resolution Reconstruction of a Single Image.' The Computer Journal 52: 15-30. doi:10.1093/comjnl/ bxm008.

[6] Drucker, H., C. J. C. Burges, L. Kauffman, A. Smola, and V. Vapnik. 1997. "Support Vector Regression Machines." In Neural Information Processing Systems 9, edited by M. C. Mozer, J. I. Joradn, and T. Petsche, 155-161. Cambridge, MA: MIT Press.

[7] Sanyal, J., and X. X. Lu. 2004. "Application of Remote Sensing in Flood Management with Special Reference to Monsoon Asia: A Review." Natural Hazards 33: 283-301. doi:10.1023/B: NHAZ. 0000037035.65105 .95

[8] Auernhammer, H. 2001. "Precision Farming-The Environmental Challenge." Computers and Electronics in Agriculture 30: 31-43. doi:10.1016/S0168-1699(00)00153-8.

[9] Gillespie, T. W., G. M. Foody, D. Rocchini, A. P. Giorgi, and S. Saatchi. 2008. "Measuring and Modelling Biodiversity from Space." Progress in Physical Geography 32: 203-221. doi:10. 1177/0309133308093606.

[10] Zhang, H., and B. Huang. 2013. "Support Vector Regression-Based Downscaling for Intercalibration of Multiresolution Satellite Images." IEEE Transactions on Geoscience and Remote Sensing 51: 1114-1123. doi:10.1109/TGRS.2013.2243736.

[11] Kim, K. I., and Y. Kwon. 2010. "Single-Image Super-Resolution Using Sparse Regression and Natural Image Prior." IEEE Transactions on Pattern Analysis and Machine Intelligence 32: 1127-1133. doi:10.1109/TPAMI.2010.25.

[12] Jiji, C. V., and S. Chaudhuri. 2006. "Single-Frame Image SuperResolution through Contourlet Learning." EURASIP Journal on Applied Signal Processing 2006: 235-235.

[13] Jiji, C. V., M. V. Joshi, and S. Chaudhuri. 2004. "Single-Frame Image Super-Resolution Using Learned Wavelet Coefficients." International Journal of Imaging Systems and Technology 14: 105112. doi:10.1002/ima.20013.

[14] Li, S., B. Yang, and J. Hu. 2011. "Performance Comparison of Different Multi-Resolution Transforms for Image Fusion." Information F[1] Loew, A., R. Ludwig, and W. Mauser. 2006. "Derivation of Surface Soil Moisture from ENVISAT usion 12 (2): 74-84. doi:10.1016/j. inffus.2010.03.002.

[15] Coops, N. C., M. Johnson, M. A. Wulder, and J. C. White. 2006. "Assessment of QuickBird High Spatial Resolution Imagery to Detect Red Attack Damage Due to Mountain Pine Beetle Infestation." Remote Sensing of Environment 103: 67-80. doi:10.1016/j.rse.2006.03.012.

[16] Hu, J., and S. Li. 2012. "The Multiscale Directional Bilateral Filter and Its Application to Multisensor Image Fusion." Information Fusion 13: 196-206. doi:10.1016/j.inffus.2011.01.002.

[17] Do, M. N., and M. Vetterli. 2005. "The Contourlet Transform: An Efficient Directional Multiresolution Image Representation." IEEE Transactions on Image Processing 14: 2091- 2106 doi:10.1109/TIP.2005.859376.

[18] Kerr, J. T., and M. Ostrovsky. 2003. "From Space to Species: Ecological Applications for Remote Sensing." Trends in Ecology and Evolution 18: 299-305. doi:10.1016/S0169-5347(03)00071-5.

[19] Da Cunha, A. L., J. Zhou, and M. N. Do. 2006. "The Non Subsampled Contourlet Transform: Theory, Design, and Applications." IEEE Transactions on Image Processing 15: 30893101. doi:10.1109/TIP.2006.877507.

[20] Milanfar, P., ed. 2010. Super-Resolution Imaging. Boca Raton, FL: CRC Press.

[21] La Rosa, D., and D. Wiesmann. 2013. "Land Cover and Impervious Surface Extraction Using Parametric and Non-Parametric Algorithms from the Open-Source Software R: An Application to Sustainable Urban Planning in Sicily." GIScience \& Remote Sensing 50 (2): 231-250.

[22] Li, M., J. Im, and C. Beier. 2013. "Machine Learning Approaches for Forest Classification and Change Analysis Using MultiTemporal Landsat TM Images over Huntington Wildlife Forest.” GIScience \& Remote Sensing 50 (4): 361-384. 
[23] Kim,Y.H.J.Im,H.K.Ha,J.K.Choi,andS.Ha.2014 "Machine Learning Approaches to Coastal Water Quality Monitoring Using GOCI Satellite Data." GIScience \& Remote Sensing 51 (2): 158174.

[24] Ishak, A. M., R. Remesan, P. K. Srivastava, T. Islam, and D. Han 2013. "Error Correction Modelling of Wind Speed through HydroMeteorological Parameters and Mesoscale Model: A Hybrid Approach." Water Resources Management 27 (1): 1-23. doi:10.1007/s11269-012-0130-1.

[25] Srivastava, P. K., D. Han, M. R. Ramirez, and T. Islam. 2013 "Machine Learning Techniques for Downscaling SMOS Satellite Soil Moisture Using MODIS Land Surface Temperature for Hydrological Application." Water Resources Management 27 (8): 3127-3144. doi:10.1007/ s11269-013-0337-9.

[26] Kim, K. I., D. H. Kim, and J. H. Kim. 2004. "Example-Based Learning for Image Super-Resolution," In Proc. 3rd TsinghuaKAIST Joint Workshop Pattern Recognition, Beijing, December 16-17, 140-148.

[27] Ni, K., and T. Q. Nguyen. 2007. "Image Superresolution Using Support Vector Regression." IEEE Transactions on Image Processing 16: 1596-1610. doi:10.1109/TIP.2007.896644.

[28] Islam, T., P. K. Srivastava, M. Gupta, X. Zhu, and S. Mukherjee eds. 2014. Computational Intelligence Techniques in Earth and Environmental Sciences, 281. Dordrecht: Springer.

[29] Rao, C.V., MalleswaraRao, J., Senthil Kumar, A., Lakshmi, B. Dadhwal, V.K.: Expansion of LISS-III swath using AWiFS wider swath data and contourlet coffecients learning. In: GI Science\& Remote Sensing, 52(1); 78-93, doi:10.1080/15481603.2014, 983370 (2015).

[30] Radhika, K.S.R., Rao, C.V., Kamakshi Prasad, V.: Enhancemen of AWiFS Spatial Resolution with SVM Learning. In 6th International Advanced Computing Conference, 978-1-4673-82861/16 IEEE DOI 10.1109/IACC.2016.42, pp. 178-183 (2016).

[31] National Remote Sensing Centre, https://bhuvan.nrsc.gov.in.

[32] Bamberger, R.H., Smith, M.J.T.: A Filter Bank for the Directional Decomposition of Images: Theory and design. In: IEEE Trans. Signal Process., vol. 40, no. 4, pp. 882-893, Ar. (1992).

[33] Xun Liu, Chenwei Deng, Baojun Zhao.: Spatiotemporal Reflectance Fusion Based on Location Regularized Sparse Representation. In: IGARSS, 978-1-5090-3332-4/16/\$31.00 C2016 IEEE, pp. 2562-2565 (2016).

[34] A Murali, K. Hari Kishore, "Efficient and High Speed Key Independent AES Based Authenticated Encryption Architecture using FPGAs "International Journal of Engineering and Technology(UAE), ISSN No: 2227-524X, Vol No: 7, Issue No: 1.5, Page No: 230-233, January 2018 